\title{
The impact of cancellations in waiting times analysis: evidence from scheduled surgeries in the Portuguese NHS
}

\author{
Joana Cima ${ }^{1}\left[\right.$ (D) Álvaro Almeida ${ }^{2}$ (i) \\ Received: 5 January 2021 / Accepted: 13 July 2021 / Published online: 25 July 2021 \\ (c) The Author(s), under exclusive licence to Springer-Verlag GmbH Germany, part of Springer Nature 2021
}

\begin{abstract}
Background Equity in access to scheduled surgery has been a topic of attention of researchers and decision-makers on healthcare. Most studies analyse the number of days that patients wait before undergoing surgery, and ignore patients that have been on the waiting list but have not benefited from surgery. This study contributes to the existing literature on waiting lists by analysing cancellations along with surgery episodes.

Methods We use a database comprising all patients that entered the waiting list for scheduled surgeries in the Portuguese National Health Service from 2011 to 2015 (around 3 million observations) and estimate survival models to explain waiting times, where cancellations are introduced as censored data.

Results The cancellation rate is significant (around 14\%), and has a considerable impact on results: ignoring cancellations biases estimates, in particular for gender differences (that are overestimated without cancelations), and for the age effect (that is underestimated).

Conclusion Thus, our approach provides a more accurate understanding of the impact that several factors have on overall access to scheduled surgery.
\end{abstract}

Keywords Scheduled surgery $\cdot$ Access $\cdot$ Cancellations $\cdot$ Survival models $\cdot$ Portugal

JEL Classification $114 \cdot \mathrm{C} 01$

\section{Introduction}

Waiting lists and waiting times for scheduled surgery are common in NHS-type systems [1]. These health systems are characterised by reduced user charges which originate excess demand and may cause long waiting times and large surgery waiting lists.

Long waiting times are a rationing mechanism constituting a barrier to access to surgical treatment [2]. There has been a wide-ranging discussion concerning equity in waiting times to scheduled surgery that focuses on patients'

Joana Cima

joanacima.phd@ fep.up.pt

Álvaro Almeida

almeida@fep.up.pt

1 Faculdade de Economia, Universidade Do Porto, 4200-464 Porto, Portugal

2 CEF.UP and Faculdade de Economia, Universidade Do Porto, Porto, Portugal characteristics (e.g., age, gender, socioeconomic status, etc.) [2-5].

Ethical considerations imply that the severity of patient's clinical condition should be the only factor able to explain differences in waiting times among patients [3]. The prioritisation for surgery process should play the central role in the formation of lists and must be able to detect the most urgent cases [6, 7]. However, other factors may play a role in the formation of waiting lists and contribute to variations in waiting times that may raise equity concerns. Identifying these factors contributes to improve the design of specific policies aimed at increasing equity and fairness in access to healthcare.

A substantial part of the literature on waiting times for scheduled surgery has focused, specifically, on patients that have been treated. These studies disregard cancellations - surgeries that were scheduled to happen but for some reason were cancelled. Cancellations may be motivated by many reasons and may have several consequences for the patient's health, both physical and emotional. They also may 
raise questions about hospital efficiency [8, 9]. A proper understanding of the barriers affecting access to surgery, and designing the adequate policies to deal with the problem, implies analysing all surgery candidates, not just the ones that eventually were submitted to surgery, and incorporate cancellations in the analysis.

Take, for instance, the spatial distribution in waiting times. If in some regions the longer waiting times are leading patients to cancel more surgeries, then the actual waiting times for surgery in those regions will be underestimated. Thus, policies implemented based on the waiting times of operated patients may be inadequate because inequalities in access are underestimated.

The inclusion of the cancelled episodes is particularly important in health systems where cancellations rates are significant. That is the case of the Portuguese National Health Service (NHS). In the Portuguese NHS, patients are registered with a primary care doctor (GP), who acts as a gatekeeper. A patient with a condition that may require surgery, must first visit her GP at the local NHS primary care health centre. The GP refers the patient to an outpatient specialist appointment at an NHS hospital (which usually occurs some months later). If the hospital specialist doctor decides that a surgery is needed, the patient is then enrolled in SIGIC, the national information system that manages the list of patients waiting surgery. If the patient does not have her surgery before the legal "guaranteed maximum time of response" (set by regulation from 3 to 270 days, depending on the patient's priority level), the patient is referred by SIGIC to either another NHS hospital or to a private hospital [10]. If the surgery becomes unnecessary before it is performed, the enrolment in SIGIC is cancelled, which was the case for $14.7 \%$ of the patients enrolled in SIGIC for the period under analysis (2011 to 2015), mostly at the initiative of the patient. ${ }^{1}$ The impact of ignoring cancellations might be large and could increase at a time when countless surgeries are cancelled or postponed due to COVID-19 [11]. It is estimated that in Portugal, between March and May 2020, about $58 \%$ of scheduled surgeries were cancelled, ${ }^{2}$ which compares with $15 \%$ in the period covered by this study. If cancellations had a relevant impact in equity in access in the period 2011-15, it is expected that analysing cancellations

\footnotetext{
1 The administrative classification of the motives for cancellation includes six categories (in parenthesis, the percentage of cancellations in our sample due to each motive): patient renunciation (37.83\%); clinical reasons (18.23\%); surgical episode that was carried out (e.g., emergency surgery) (9.38\%); transfer to another hospital (8.05\%); patients' death $(2.11 \%)$; other reasons (24.40\%). Additional information will be made available upon request.

2 Assuming a scenario without COVID-19 where the values between March and May 2020 would be the same as in 2019. Source: Portal da Transparência do SNS—https://www.sns.gov.pt/transparencia/.
}

will be even more crucial in the COVID-19 period, when cancellation rates almost quadrupled.

Thus, this paper revises the way the topic of equity in access to scheduled surgery has been addressed. We use information on all exits of waiting lists, including surgical procedures performed along with cancellations, to study equity in access to scheduled surgery. This study uses information on all exits of waiting lists for scheduled surgeries on the Portuguese NHS between 2011 and 2015 (around 3 million observations), to estimate survival models that include censored data associated with cancellations and assess the impact on results of including, or not, cancellation episodes.

\section{Background}

Cancellations for surgical treatment correspond to patients who left the waiting list before being subjected to surgery. Cancellations are an important feature of waiting lists: $\mathrm{Al}$ Talalwah and Mclltrot [12] developed a literature review on cancellations for surgery and found cancellations rates ranged between 5 and 39\%.

Although the literature on cancellations is vast, a substantial part of these studies has focused on statistical analysis of specific clinical conditions and/or motivations for cancelling [13-19].

On the other hand, the literature on waiting times to scheduled treatment is focused on operated patients. See, for instance, the studies of Laudicella et al. [3], Cooper et al. [4], Johar et al. [20], Landi et al. [2] that evaluated the factors besides the severity of the patient's clinical condition that might influence access to treatment. The authors showed that patients' specific socioeconomic and demographic characteristics influence waiting times. Laudicella et al. [3] found that waiting times for patients admitted for hip replacement in the English NHS are shorter for the elderly and that men have longer waiting times compared with women. An identical finding was obtained by Cooper et al. [4] for patients submitted to hip replacements. On the contrary, Johar et al. [20] observed longer waiting times for older patients, and also reported that men wait less time than women in New South Wales (Australia). Landi et al. [2] concluded that gender and age were not relevant in explaining excessive waiting times for elective surgery in the Italian NHS. Also, Laudicella et al. [3], Johar et al. [20] and Landi et al. [2] found waiting times favour the most socioeconomic advantaged patients.

An open question in this literature is whether results on inequalities would be the same if cancellations were added. As noted earlier, cancellations do not refer to completed observations because they are associated with premature exits from the waiting list. Thus, those observations can be understood as censored data that take place when "incomplete information is available about the survival time of 
Table 1 Distribution of the waiting times by surgery and cancellation

\begin{tabular}{lcc}
\hline Days & Surgery & Cancellation \\
\hline$<=1$ & $188,413(7 \%)$ & $17,073(4 \%)$ \\
] $1-30]$ & $995,476(37 \%)$ & $83,584(18 \%)$ \\
]30-90] & $736,387(27 \%)$ & $86,815(19 \%)$ \\
$>90$ & $770,454(29 \%)$ & $278,966(60 \%)$ \\
TOTAL & $2,690,730(100 \%)$ & $466,438(100 \%)$ \\
\hline
\end{tabular}

some individuals" [21] or "a subject in the study withdraws prematurely" [22]. Focusing on completed observations and dropping censored episodes is, in fact, a standard procedure when one is in the presence of censored data. However, this procedure can lead to sample selection problems and produce inconsistent estimators $[21,23]$ if we aim at understanding the impact on waiting times for all scheduled surgeries.

McIntosh et al. [24], Cookson et al. [25] and Cookson et al. [8] are part of the reduced literature that analysed cancellations along with surgery episodes. The authors evaluated which factors contributed to last-minute cancellations in scheduled surgery in the English NHS and employed binary outcome models with the dependent variable coded according to the cancelled or operated event. For instance, McIntosh et al. [24] and Cookson et al. [25] found that patients from lower socioeconomic groups, men and older patients have higher probability of cancellation.

However, their objective was merely to look at factors affecting the decision to cancel surgery. Our study, on the other hand, analyses the impact of a set of variables on the time-to-event (surgery) adjusting for censored observations (cancellations) using survival models [22]. Thus, our study improves on the understanding of which factors impact on waiting time to surgery and on the inequalities in access that may exist, by reducing the selection bias that may arise from the exclusive use of episodes of performed surgeries.

\section{Data and methodology}

\section{Data}

This work is based on the administrative data of the list for all scheduled surgeries in the Portuguese NHS from 2011 to $2015 .^{3}$ The database includes episodes of surgery and cancellation episodes as well. The waiting times correspond to the period elapsed from the moment of entry in the list until the patient has been operated or has left the list by cancellation.

\footnotetext{
3 The administrative data were obtained from SIGIC, provided by the Portuguese Central Administration of the Health System (ACSS).
}

Table 1 shows the distribution of waiting times, both for surgeries performed and for episodes of cancellation. Episodes of surgery are more frequent in the first month of entry into the list, but cancellation frequency is higher for longer waiting times.

Table 2 presents the distribution of surgeries and cancellations by patient's priority, and the rules for waiting times according to priority to be applied by the NHS. ${ }^{4}$ One can observe that the cancellation rate is higher for priorities considered to be less severe, as expected, since higher priorities require a timelier surgical treatment. However, both priority levels 3 and 4 still have a cancellation rate of around $8 \%$.

Figure 1 displays the plot of Kaplan-Meier survival estimates. The vertical axis corresponds to the estimated probability of survival occurring within a specific time (for all individuals in our sample), and the horizontal axis is the number of days from the moment of entry into the list. ${ }^{5}$ The likelihood of survival occurrence starts, as expected, from one and decreases to zero where the highest reduction is obtained in first weeks. However, it is clear that waiting times extend well beyond the time limits in the official rules, and that they differ substantially among patients. It is then essential to understand to what extent the characteristics of patients have affected access to surgery.

Table 3 provides descriptive statistics that allow for a characterization of the differences between the waiting times of operated patients and of cancellations.

\section{Survival model}

We estimate survival models for patients in the surgery list that simultaneously account for patient individual characteristics such as gender, age, priority level and cancer indicator, while introducing several additional controls that account for other sources of heterogeneity such as hospital, speciality, surgical procedure or place of residence. Age was categorized in six groups: below 15 years old; 15 to $29 ; 30$ to 44 ; 45 to 59; 60 to 74; above 75 years old. Dummy variables for the speciality and surgical procedures were included to account for the specificities of surgical treatment. ${ }^{6}$ Hospital dummies capture differences in waiting times due to the organisational structure or other factors which may be specific to each hospital. Dummy variables for patient's place of

\footnotetext{
${ }^{4}$ The priority level is assigned considering the maximum time that the patient can wait for surgery. It takes into account the disease and associated problems, pathology, severity, impact on life expectancy, autonomy and quality of life, speed of disease progression and time of exposure to the disease [26].

5 We restrict the graph to $t<=365$ days to simplify the analysis.

${ }^{6}$ Since there are thousands of categories on surgical procedures, one cannot control for all the procedures categories due to computational constraints. Dummy variables were used for the 300 most common surgical procedures, and the rest was coded as "others".
} 
Table 2 Distribution of surgery and cancellation - by priority

\begin{tabular}{|c|c|c|c|c|}
\hline Priority & Description $^{\mathrm{a}}$ & Surgery & Cancellation & $\begin{array}{l}\text { Cancella- } \\
\text { tion rate } \\
(\%)\end{array}$ \\
\hline 1 & $\begin{array}{l}\text { Waiting time up to } 270 \text { days for } \\
\text { the surgery, or } 60 \text { days in the case } \\
\text { of an oncological disease }\end{array}$ & $2,038,355$ & 399,232 & 16.4 \\
\hline 2 & $\begin{array}{l}\text { The surgical treatment cannot exceed } \\
\text { more than } 60 \text { days or } 45 \text { days } \\
\text { in case of an oncological disease }\end{array}$ & 433,919 & 48,476 & 10.1 \\
\hline 3 & $\begin{array}{l}\text { Surgery has to be carried out } \\
\text { within a maximum of } 15 \text { days }\end{array}$ & 130,282 & 11,144 & 7.9 \\
\hline 4 & $\begin{array}{l}\text { Surgery has to be performed } \\
\text { within a maximum of } 3 \text { days or } \\
\text { during the patient's hospitalisation }\end{array}$ & 88,174 & 7,586 & 7.9 \\
\hline
\end{tabular}

${ }^{a}$ Diário da República[27]

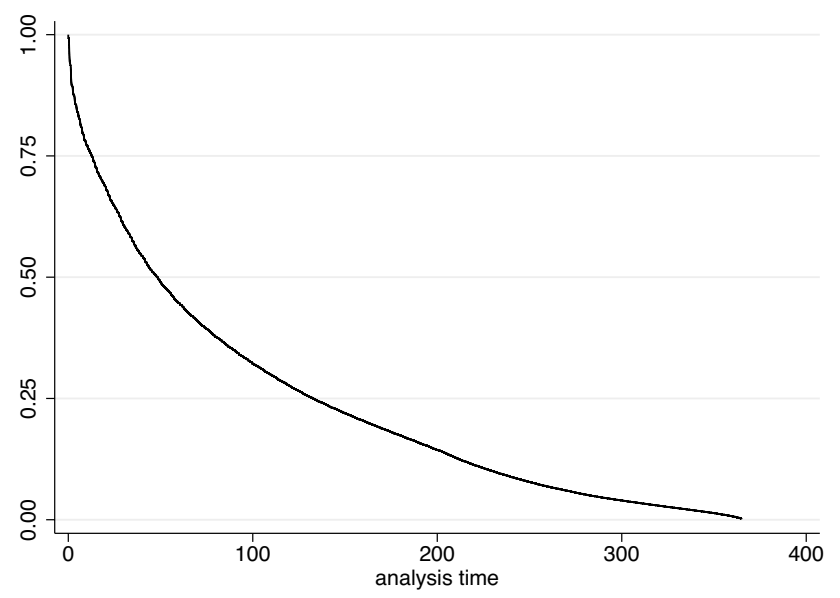

Fig. 1 Kaplan-Meier survival estimate

residence (municipality) capture regional disparities, such as average income, education level, or access to health services that may impact waiting times. Finally, the variable "year" was included to allow for changes in nationwide policy decisions that may affect all waiting times.

The survival models to be estimated are proportional hazard models, where surgery is the event of interest and cancellation corresponds to censored observations. The general specification is given by:

$h(t)=h_{0}(t) \times \exp (X \beta)$

where $h(t)$ is the hazard rate of the surgery, also known as the instantaneous rate of an event occurrence, $\mathrm{X}$ corresponds to a vector of covariates (gender, age, priority, cancer, speciality, procedure code, year, hospital, municipality of residence), $\beta$ are the coefficients to be estimated and $h_{0}(t)$ is the baseline hazard.
The parameters $\beta$ indicate how the risk (likelihood) of surgery increases/decreases according to the degree of exposure. For instance, if $\beta_{\text {male }}>1$, one may conclude that, conditional on all other variables, men are more likely to be submitted to surgery, and therefore, they have lower waiting times than women.

The baseline hazard function $h_{0}(t)$ may be modelled using different specifications. We estimate the Weibull model, a popular parametric method in survival analysis $[22,28]$, that assumes $\mathrm{h}_{0}(\mathrm{t})=\rho \mathrm{t}^{\rho-1} \exp \left(\beta_{0}\right)$ [22]. Imposing a Weibull specification without further considerations could be too restrictive since its main limitation lies in the hazard monotonicity [28]. However, this is not the case for our model, since alternative specifications yield similar results. ${ }^{7}$ The Weibull model is estimated by maximum likelihood where the log likelihood function to be maximised is based on the non-informative censoring assumption (meaning that it assumes the censoring times are independent of the failure times conditional on values of covariates) [29, 30].

Although treating informative censoring as non-informative may bias the estimates, it is usually recognised the difficulty on identifying informative censoring and testing its impact on results [21,31]. Therefore, since one cannot exclude the presence of informative censoring, we performed a sensitivity analysis to observe the impact of different survival times on estimates. We use the best and

\footnotetext{
7 We have also estimated a Piecewise exponential model, that accounts for non-monotone hazards splitting time into different intervals and assuming the hazard rate is constant within each one, but that it may vary between intervals. We have also estimated a Cox model where $h_{0}(\mathrm{t})$ is an unknown nonnegative function. Both models provide very similar results to those of the Weibull model. In the particular case of the PWE, the results reveal a similar monotonicity to that of the Weibull model with regard to $h_{0}(\mathrm{t})$. Weilbull's distribution also has the property of being parameterized either with proportional hazards or with accelerate failure time.
} 
Table 3 Descriptive statistics of waiting times (WT) for surgery and cancellation

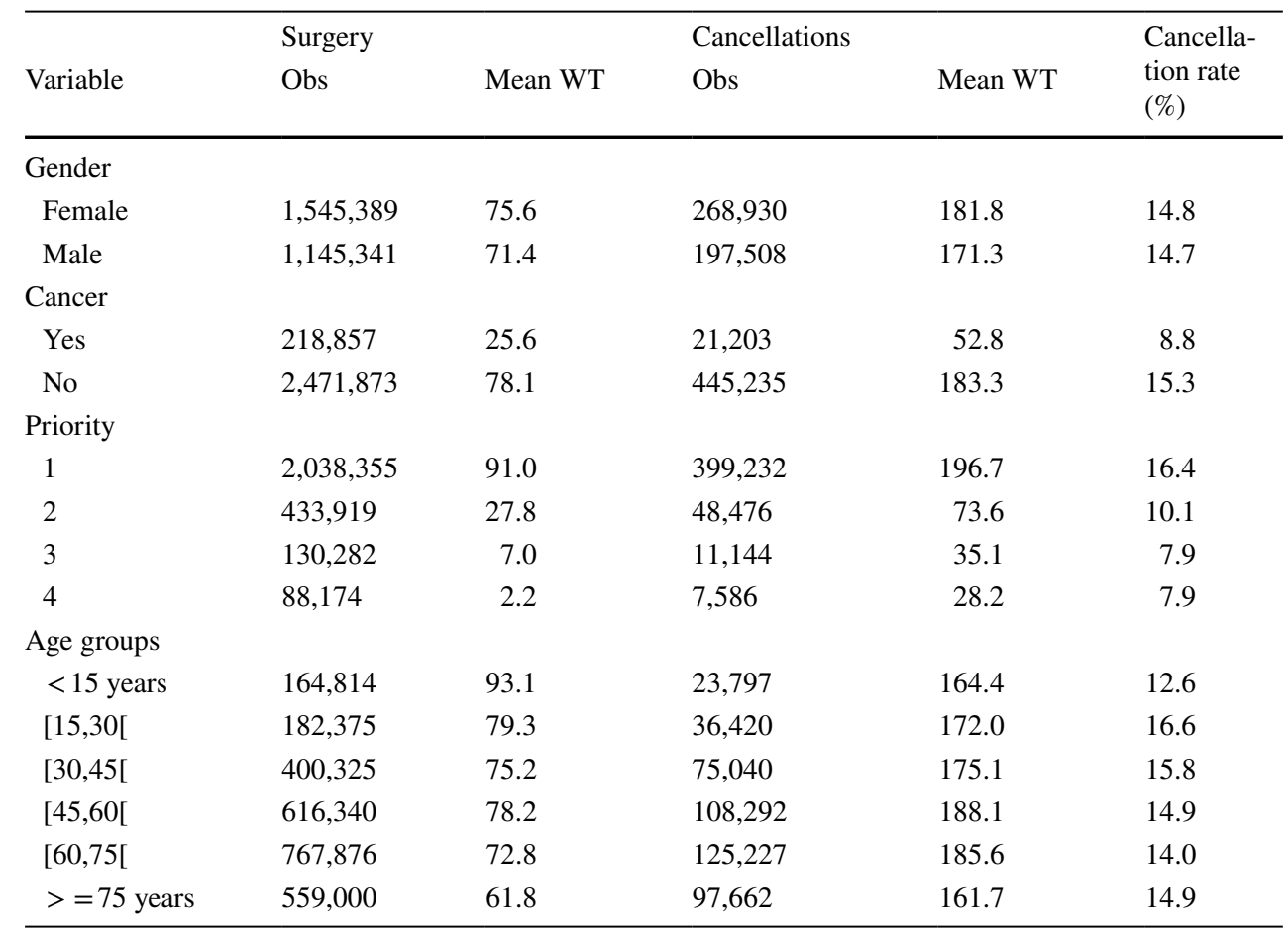

the worst scenarios approach, wherein, first we assume that censored data are non-informative, second, we consider an extreme relationship between the censored data and the hazard rate by assuming that all censored patients have surgery at the time they have cancelled [31]. If the estimates change considerably, it means that not correcting the likelihood function for the presence of informative censoring can have strong implications in the results. However, if the conclusions do not change, this means that even in the presence of informative censoring results are not particularly affected.

Finally, to infer how the inclusion of cancellations impacts the results, we also estimate the model using only observations for patients submitted to surgery (without cancellations). If there are relevant differences between the two estimates, with and without cancellations, one may conclude that it is essential to include cancellations in waiting lists studies, because the results on access are biased when using operated patients only.

\section{Results}

Table 4 reports the main results of the estimation of the model. Column 1 corresponds to the estimation where surgery is the event of interest and cancellation is the censored event, assuming non-informative censoring. Column 2 presents the results of the extreme sensitivity analysis to observe the effect that informative censoring has on results. As expressed in the previous section, in the worst-case scenario, all censored patients are estimated as if they had been operated at the time of cancellation. Column 3 displays the results of the estimation of the model not including the cancellations. $^{8}$

In the three models, estimations show that prioritization levels, as well as the cancer indicator, are the variables that most influence the waiting times for surgery. Waiting times decrease with more severe priorities, with priority 4 having the shortest waiting times, as expected. Patients with cancer have shorter waiting times than patients not reported to have cancer. Men are more likely to be operated, and consequently, have shorter waiting times than women. The age group from 30 to 45 years (reference group) is the one with the shortest waiting times.

Looking at column 1 we find the hazard for surgery is 12 times higher for patients with the most severe priority compared with priority 1 . Patients reported with cancer have 1.47 higher hazards than the base group while the hazard for men is 1.02 higher compared with women. The age group above 74 years has a 0.90 lower hazard than the reference group and therefore has longer waiting times. The coefficients for the models in columns 2 and 3 should be interpreted in a similar fashion. The shape of the Weibull function shows a negative duration dependence $\rho<1$. Thus, the hazard function is monotonically decreasing indicating that the probability of surgery decreases over time. The fact that the $95 \%$ confidence interval for $\rho$ does not include the value

\footnotetext{
8 The Appendix shows the estimation of a logit model that helps understand the determinants for cancellation.
} 
Table 4 Estimation of the duration Weibull models

\begin{tabular}{|c|c|c|c|}
\hline & (1) & $\begin{array}{l}\text { (2) } \\
\text { Informative censoring }\end{array}$ & (3) \\
\hline & Non-1niormative censoring & inrormative censoring & Operated patients oniy \\
\hline Constant & $0.2511 * * *$ & $0.2135 * * *$ & $0.3152 * * *$ \\
\hline \multicolumn{4}{|l|}{ Gender } \\
\hline Male & $1.0245 * * *$ & $1.0298 * * *$ & $1.0365 * * *$ \\
\hline \multicolumn{4}{|l|}{ Cancer } \\
\hline Yes & $1.4646 * * *$ & $1.4354 * * *$ & $1.3533 * * *$ \\
\hline \multicolumn{4}{|l|}{ Priority } \\
\hline 2 & $2.2422 * * *$ & $2.1847 * * *$ & $2.2642 * * *$ \\
\hline 3 & $6.1818 * * *$ & $5.9637 * * *$ & $6.7989 * * *$ \\
\hline 4 & $11.6622 * * *$ & $11.4302 * * *$ & $15.877 * * *$ \\
\hline \multicolumn{4}{|l|}{ Age } \\
\hline$<15$ years & $0.9059 * * *$ & $0.8916^{* * *}$ & $0.8603 * * *$ \\
\hline$[15-30[$ & $0.9581 * * *$ & $0.9626 * * *$ & $0.9628 * * *$ \\
\hline$[45-60[$ & $0.9655^{* * *}$ & $0.9646 * * *$ & $0.9650 * * *$ \\
\hline$[60-75[$ & $0.9482 * * *$ & $0.9597 * * *$ & $0.9716^{* * *}$ \\
\hline$>=75$ years & $0.9005^{* * *}$ & $0.9496 * * *$ & 0.9986 \\
\hline$\rho$ & 0.7898 & 0.8240 & 0.8763 \\
\hline $\mathrm{N}$ & $3,156,956$ & $3,156,956$ & $2,690,554$ \\
\hline Wald & $669,527.20 * * *$ & $666,407.50 * * *$ & $848,965.25^{* * *}$ \\
\hline Log pseudolikelihood & $-5,407,195.6$ & $-5,638,551.5$ & $-4,650,420.4$ \\
\hline
\end{tabular}

*** $p<0.01$. The regressions include dummy variables for year, hospital, municipality, speciality and procedure code, whose results are not displayed in this table but are available upon request

1 (in the three models) is an indication that the Exponential model is not a good fit for the data.

The results in column 2 , for the model that assumes the extreme scenario that all censored data are informative, are qualitatively similar to the results in column 1 , for the model that assumed the censored data are non-informative, although there are some quantitative differences. This suggests that informative censoring does not seem to have a major impact on results. Nevertheless, informative censoring in the extreme case implicit in the Column 2 estimation (all censored patients have surgery in the time they have cancelled) is unlikely to be relevant in this context, since patients that cancel are unlikely to have surgery.

Column 3 shows that excluding cancellations biases the results in a dimension that affects the conclusions of the analysis. The coefficients for priority are much larger in the model estimated without cancellations, suggesting that the impact of these variables may be overestimated, especially in the case of priority 4 , with estimates of the impact of this variable on waiting times that are about $40 \%$ higher. On the other hand, the effect of the cancer indicator is $24 \%$ undervalued.

The bias introduced by the omission of the cancellations is also observable in the results for the control variables. Figure 2 shows the hazard ratios distribution across the municipalities of mainland Portugal: the map on the left shows the distribution of the hazard ratios obtained in the model with censored data; the map on the right shows the coefficients provided by the model with data only on operated patients. The upper quartiles refer to the municipalities with the highest hazard ratios and, therefore, the shortest waiting times.

Table 5 shows the hazard ratios distribution regarding hospital, speciality, procedure code, and municipality using the two estimation strategies. The last column shows the percentage of categories within each variable that change quartiles, by applying the two methods. For example, 26.2\% of hospitals change their distribution quartile according to the estimation strategy.

\section{Discussion}

The previous section shows that results differ substantially with the inclusion of cancellations, which implies that drawing conclusions about those factors that impact on waiting times based only on the subsample of patients submitted to surgery would exclude valuable information and might not correctly inform decision-makers.

The most important differences regard the gender and age bias that previous literature has found in waiting lists. Johar et al. [20], and Moscelli et al. [32] have found a gender bias, with men having lower waiting times to surgery. Our results confirm the existence of the bias but show that the estimates 

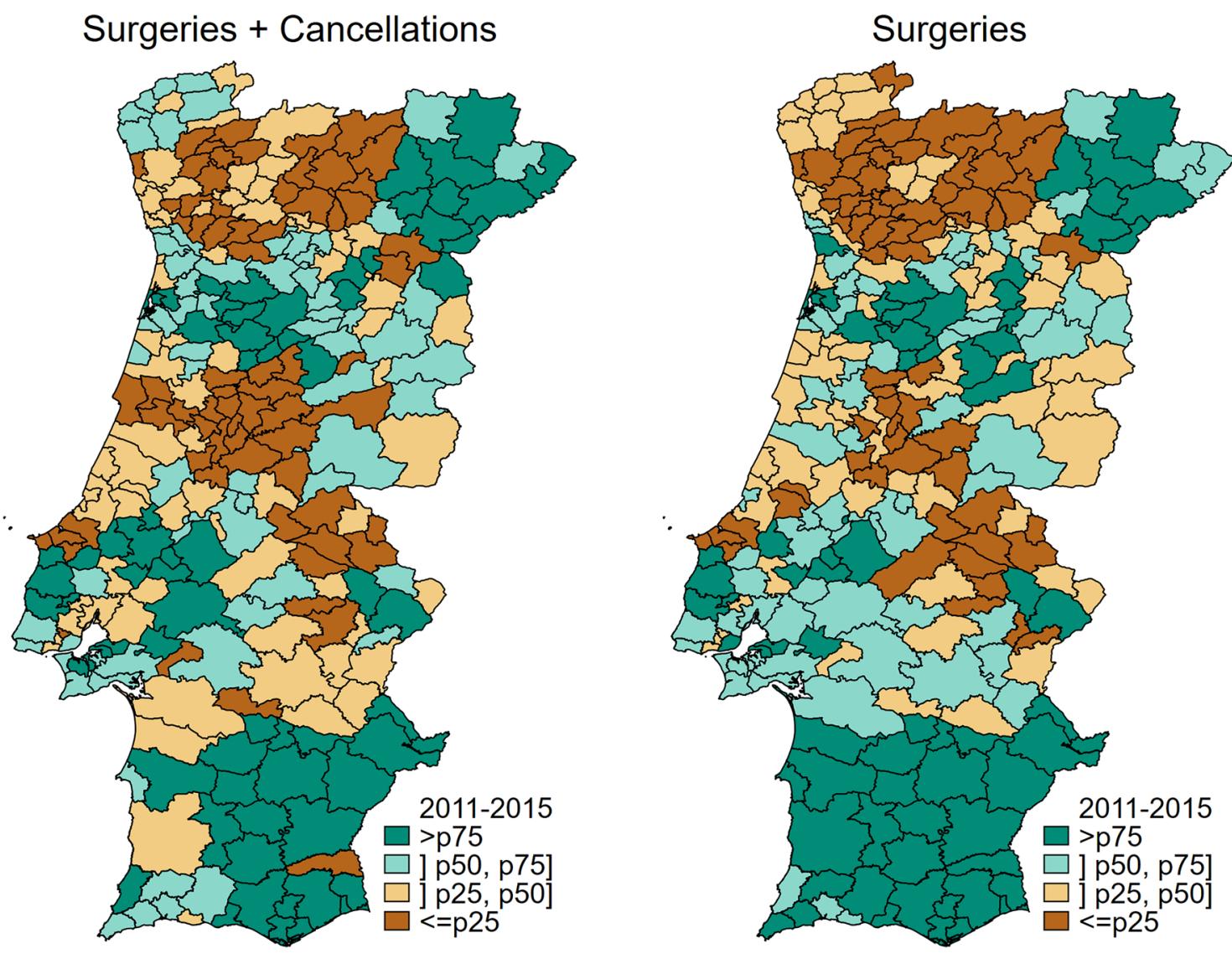

Fig. 2 Hazard ratios across the Portuguese municipalities

Table 5 Hazard ratios distribution for Hospital, Speciality, Procedure Code and Municipality

\begin{tabular}{|c|c|c|c|c|c|c|c|}
\hline \multirow[t]{2}{*}{ Variable } & \multicolumn{3}{|c|}{ Surgeries + Cancellations } & \multicolumn{3}{|c|}{ Surgeries } & \multirow{2}{*}{$\begin{array}{l}\% \text { quartile } \\
\text { changes }\end{array}$} \\
\hline & p25 & p50 & p75 & p25 & p50 & p75 & \\
\hline Hospital & 0.4797 & 0.5714 & 0.7115 & 0.4704 & 0.5687 & 0.7060 & 26.2 \\
\hline Speciality & 0.1440 & 0.1794 & 0.2505 & 0.0915 & 0.1168 & 0.1659 & 7.3 \\
\hline Procedure code & 1.0167 & 1.2112 & 1.5488 & 1.0929 & 1.3287 & 1.6896 & 20.6 \\
\hline Municipality & 0.9471 & 0.9875 & 1.0397 & 0.9201 & 0.9604 & 1.0232 & 31.8 \\
\hline
\end{tabular}

of the bias are exaggerated when cancellations are ignored. The model estimated without cancellations produces an estimate of the gender bias $(3.7 \%)$ that is $50 \%$ higher than the more accurate estimation of the model that considers all patients that needed surgery, including those that were not submitted to surgery $(2.5 \%)$. This result is consistent with the literature that shows men have a lower rate of health care utilization $[33,34]$, which may reflect in cancelling the scheduled surgery earlier than women; in this case, some women have surgery after waiting for a longer time, while men that could have surgery at the same time were not submitted to surgery because they had previously cancelled it.

The more accurate estimation of the model with cancellations identifies a significant age bias, with waiting times increasing with age for patients older than 45, a trend that is not clear in the model without cancellations. The age group from 30 to 45 years (reference group) is the one with the shortest waiting times and as age increases the probability of surgery decreases, causing a rise in waiting times. This result is consistent with Johar et al. [20] regarding the impact of age on waiting times, that is, older patients have longer waiting times. Since cancellations tend to increase with age, as McIntosh et al. [24] and Cookson et al. [25] have shown (in their paper age above 60 years was relevant in explaining cancellations and the effect got stronger as age increased), older patients are less likely to have surgery after waiting for a long time. A possible explanation may have to do with 
less ability to pressure medical staff and express dissatisfaction with longer waiting times, resulting from lower qualifications, or technological barriers. This implies that younger patients will have more surgeries with long waiting times in the surgeries data, increasing their average waiting times, even though older patients effectively have to wait longer. In our estimates, ignoring the cancellations prevents researchers and policy makers of identifying the discrimination of older patients.

The ranking of hospitals in terms of hazard ratios (and thus, waiting times) is substantially different when cancellations are excluded from the analysis (26\% of hospitals change to a different quartile). The result of hospitals is related to the quality or type of hospital (e.g., university hospitals) that may impact waiting times and cancellations. For example, McIntosh et al. [24] found that provider characteristics were important to explain cancellations.

Similar differences in results were found for the ranking of procedure codes, but not for the ranking of specialities, for which differences in results with and without cancellations were much smaller (only $7 \%$ of specialities change quartiles without cancellations). This result is probably due to a homogeneous pattern of cancellations for each speciality, that could be determined by the availability of resources (material or human resources) in each speciality. Cancellations are likely to be higher for those specialities for which the resources available in the NHS are lower relative to the alternatives available outside the NHS that patients may opt to seek.

Differences in the hazard ratio for municipalities are likely to reflect differences in access to hospitals (e.g., travelling distance) and socioeconomic differences. There are major differences across municipalities in Portugal regarding income levels, unemployment rates, population benefiting from social security payments, education levels, and other socioeconomic variables. Since the dataset used does not include information on these variables for individual patients, the impact of socioeconomic variables on waiting times is likely to be captured by the variable Municipality. Patients from municipalities with lower income or which are more distant from hospitals may have a higher probability of cancellation (at least for non-clinical reasons) since geographical barriers are recognized to limit access to healthcare in Portugal [10].
Nevertheless, the precise explanation of differences in waiting times for these variables is a matter that requires further investigation that is beyond the scope of this paper. What seems clear from our research is that drawing conclusions about those factors that impact waiting times based only on the subsample of patients submitted to surgery would exclude valuable information and might not correctly inform decision-makers.

\section{Conclusion}

This study analyses equity in access to surgery, using all available information, which includes patients that were submitted to surgical procedures along with patients that were scheduled for surgery but did not have the surgery, due to cancellation. We use data from all patients that exit the waiting list for scheduled surgery in the Portuguese NHS between 2011 and 2015, to estimate survival models with episodes of surgery along with cancellations.

We show that models of waiting times that are estimated only with patients that were submitted to surgery present a biased picture of the factors affecting waiting times. We found two important policy implications of these biases. First, the gender bias identified in the literature (men have shorter waiting times) is much lower than estimated by models without cancellations, implying that policymakers might employ more resources than necessary in policies directed at eliminating the discrimination of women. Second, there is a significant age bias (older patients have to wait longer) that was not identified by the model without cancellations, implying that policymakers might neglect an important lack of equity in access to scheduled surgeries.

\section{Appendix}

Table 6 shows the estimation of a logit model that helps explain the probability of cancellation. Although this analysis is not the focus of the paper, there are some results to be pointed out. The estimates show that men are more likely to cancel compared to women. Patients with more severe priorities, as well as patients reported with cancer, are less likely to cancel. Regarding age, it is observed that older patients have an increased probability of cancellation. 
Table 6 Estimation of logit model

\begin{tabular}{ll}
\hline & Logit (cancellation) \\
\hline Constant & $0.0099 * * *$ \\
Gender & \\
Male & $1.0281 * * *$ \\
Cancer & \\
Yes & $0.6682 * * *$ \\
Priority & \\
2 & $0.6085^{* * *}$ \\
3 & $0.3925 * * *$ \\
4 & $0.3827 * * *$ \\
Age & \\
$<15$ years & $0.9053 * * *$ \\
[15-30[ & $1.0349 * * *$ \\
[45-60[ & 1.0007 \\
$>=75[$ & $1.0983 * * *$ \\
$\mathrm{~N}$ & $1.5459 * * *$ \\
Wald & $3,156,668$ \\
Log pseudolikelihood & $126,450.18 * * *$ \\
\hline
\end{tabular}

*** $p<0.01$. The regressions include dummy variables for year, hospital, municipality, speciality and procedure code, whose results are not displayed in this table but are available upon request

Acknowledgements The authors thank Prof Paulo Guimarães for his valuable contribution to the paper. Joana Cima acknowledges the financial support from the Portuguese Science Foundation (FCT) in the framework of the PhD Scholarship: SFRH/BD/115954/2016.

Availability of data and material Portuguese Central Administration of Health System (ACSS) owns administrative data analysed in the paper. Therefore, data cannot be made publicly accessible.

\section{Declarations}

Conflict of interest The authors declare no conflict of interest.

\section{References}

1. Siciliani, L., Hurst, J.: Tackling excessive waiting times for elective surgery: a comparative analysis of policies in 12 OECD countries. Health Policy 72(2), 201-215 (2005)

2. Landi, S., Ivaldi, E., Testi, A.: Socioeconomic status and waiting times for health services: An international literature review and evidence from the Italian National Health System. Health Policy 122(4), 334-351 (2018)

3. Laudicella, M., Siciliani, L., Cookson, R.: Waiting times and socioeconomic status: evidence from England. Soc Sci Med 74(9), 1331-1341 (2012)

4. Cooper, Z.N., McGuire, A., Jones, S., Grand, J. Le: Equity, waiting times, and NHS reforms: retrospective study. BMJ 339 (2009)

5. Cima, J., Guimarães, P., Almeida, A.: Explaining the gender gap in waiting times for scheduled surgery in the Portuguese National
Health Service. Port J Public Health 39, 3-10 (2021). https://doi. org $/ 10.1159 / 000514798$

6. Gravelle, H., Siciliani, L.: Is waiting-time prioritisation welfare improving? Health Econ 17(2), 167-184 (2008)

7. Askildsen, J.E., Holmas, T.H., Kaarboe, O.: Prioritization and patients' rights: analysing the effect of a reform in the Norwegian hospital sector. Soc Sci Med 70(2), 199-208 (2010)

8. Cookson, G., Jones, S., Laliotis, I.: Cancelled procedures in the English NHS: Evidence from the 2010 tariff reform. Health Econ 26(12), e126-e139 (2017)

9. Hovlid, E., Bukve, O., Haug, K., Aslaksen, A.B., von Plessen, C.: A new pathway for elective surgery to reduce cancellation rates. BMC Health Serv Res 12(1), 154 (2012)

10. Simões, J., Augusto, G.F., Fronteira, I., Hernández-Quevedo, C.: Portugal: Health system review. Health Syst in Transit 19(2), 1-184 (2017)

11. CovidSurg Collaborative: Elective surgery cancellations due to the COVID-19 pandemic: global predictive modelling to inform surgical recovery plans. Br J Surg (2020)

12. Al Talalwah, N., McIltrot, K.H.: Cancellation of surgeries: Integrative review. J Perianesth Nurs 34(1), 86-96 (2018)

13. Dimitriadis, P.A., Iyer, S., Evgeniou, E.: The challenge of cancellations on the day of surgery. Int J Surg 11(10), 1126-1130 (2013)

14. Chang, J.H., Chen, K.W., Chen, K.B., Poon, K.S., Liu, S.K.: Case review analysis of operating room decisions to cancel surgery. BMC Surg 14, 47-47 (2014)

15. Ezike, H.A., Ajuzieogu, V.O., Amucheazi, A.O.: Reasons for elective surgery cancellation in a referral hospital. Ann Med Health Sci Res 1(2), 197-202 (2011)

16. Kumar, R., Gandhi, R.: Reasons for cancellation of operation on the day of intended surgery in a multidisciplinary 500 bedded hospital. J Anaesthesiol Clin Pharmacol 28(1), 66-69 (2012)

17. Bamashmus, M., Haider, T., Al-Kershy, R.: Why is cataract surgery canceled? a retrospective evaluation. Eur J Ophthalmol 20(1), 101-105 (2010)

18. Sanjay, P., Dodds, A., Miller, E., Arumugam, P.J., Woodward, A.: Cancelled elective operations: an observational study from a district general hospital. J Health Organ Manag 21(1), 54-58 (2007)

19. Caesar, U., Karlsson, J., Olsson, L., Samuelsson, K., HanssonOlofsson, E.: Incidence and root causes of cancellations for elective orthopaedic procedures: a single center experience of 17,625 consecutive cases. Patient Saf Surg 8(1), 24 (2014)

20. Johar, M., Jones, G., Keane, M.P., Savage, E., Stavrunova, O.: Discrimination in a universal health system: explaining socioeconomic waiting time gaps. J Health Econ 32(1), 181-194 (2013)

21. Leung, K.M., Elashoff, R.M., Afifi, A.A.: Censoring issues in survival analysis. Annu Rev Public Health 18, 83-104 (1997)

22. Cleves, M, Gould, W., Marchenko, Y.: An Introduction to Survival Analysis Using Stata. Stata Press, revised third edition (2016)

23. Wooldridge, J.M.: Corner Solution Outcomes and Censored Regression Models. The MIT Press Cambridge, London, England (2002)

24. McIntosh, B., Cookson, G., Jones, S.: Cancelled surgeries and payment by results in the English National Health Service. J Health Serv Res Policy 17(2), 79-86 (2012)

25. Cookson, G., Jones, S., McIntosh, B.: Cancelled procedures: inequality, inequity and the National Health Service reforms. Health Econ 22(7), 870-876 (2013)

26. Diário da República: Portaria n.25/2008 de 15 de janeiro (2008)

27. Diário da República: Portaria n.87/2015 de 23 de março (2015)

28. Lai, C.: Weibull distribution. In Generalized Weibull Distributions, Springer, Berlin (2014)

29. O'Quigley, J.: Proportional Hazards Regression. Statistics for Biology and Health, Springer-Verlag, New York (2008) 
30. Sianni, F., Copas, J.: Sensitivity analysis for informative censoring in parametric survival models. Biostatistics 6(1), 77-91 (2005)

31. Clark, T.G., Bradburn, M.J., Love, S.B., Altman, D.G.: Survival analysis part iv: Further concepts and methods in survival analysis. Br J Cancer 89, 781-786 (2003)

32. Moscelli, G., Siciliani, L., Gutacker, N., Cookson, R.: Socioeconomic inequality of access to healthcare: Does choice explain the gradient? J Health Econ 57, 290-314 (2018)

33. Perelman, J., Fernandes, A., Mateus, C.: Gender disparities in health and healthcare: results from the Portuguese National Health Interview survey. Cad Saude Publica 28(12), 2339-2348 (2012)
34. Bertakis, K.D., Azari, R., Helms, L.J., Callahan, E.J., Robbins, J.A.: Gender differences in the utilization of health care services. J Fam Pract 49(2), 147-147 (2000)

Publisher's Note Springer Nature remains neutral with regard to jurisdictional claims in published maps and institutional affiliations. 